\title{
DEEP SEARCHES FOR RADIO PULSATIONS AND BURSTS FROM FOUR SOUTHERN ANOMALOUS X-RAY PULSARS
}

\author{
Fronefield Crawford, ${ }^{1}$ Jason W. T. Hessels, ${ }^{2,3}$ and Victoria M. Kaspi ${ }^{2}$ \\ Received 2006 December 14; accepted 2007 March 9
}

\begin{abstract}
We have searched for persistent radio pulsations, bright single pulses, and bursts from four southern anomalous X-ray pulsars (AXPs). Deep observations were conducted at $1.4 \mathrm{GHz}$ in 1999 July and August with the Parkes $64 \mathrm{~m}$ telescope. For all of the target AXPs, the upper limits on integrated pulsed emission are $\sim 0.02 \mathrm{mJy}$, while the limits on the flux of single pulses are $\sim 1 \mathrm{Jy}$ or better. The corresponding radio luminosity limits are significantly below the observed luminosities of the majority of the observed radio pulsars, and they are significantly below the radio luminosity of XTE J1810 - 197, the only AXP known thus far to emit radio pulsations, at the epoch when radio pulses were first detected from this source. Our null results support the hypothesis that pulsed radio emission from AXPs is only present in conjunction with X-ray outburst activity. However, we cannot rule out the possibility that pulsed radio emission is present, but that it is too weak to be detected in our observations, or that unfavorable viewing geometries prevent this emission from being seen by terrestrial observers. Given the possible association between the radio emission and transient X-ray behavior of XTE J1810-197, continued radio searches of these and other AXPs at different epochs are warranted, particularly after periods of X-ray burst activity.
\end{abstract}

Subject headings: pulsars: general — stars: neutron

\section{INTRODUCTION}

Anomalous X-ray Pulsars (AXPs), thought to be magnetars (see Woods \& Thompson 2006; Kaspi 2007 for a review), are a rare class of slowly rotating $\left(P_{\text {spin }}=5-12\right.$ s) neutron stars, whose $\mathrm{X}$-ray emission may be powered by the decay of their huge inferred magnetic fields $\left(B_{\text {surf }}=10^{14}-10^{15} \mathrm{G}\right)$. Although they are relatively bright X-ray sources $\left(10^{34}-10^{36} \mathrm{erg} \mathrm{s}^{-1}\right)$, previous attempts to detect radio pulsations from these objects have been unsuccessful (e.g., Israel et al. 2002; Crawford et al. 2002; Burgay et al. 2006b). Recently, however, Camilo et al. (2006b) reported the discovery of pulsed radio emission from the transient AXP XTE J1810-197 (Ibrahim et al. 2004) in 2006 March, subsequent to the discovery of a radio point source in VLA imaging of the area (Halpern et al. 2005). These discoveries have provided an exciting new spectral window on magnetar physics and another link between the AXPs and conventional, rotation-powered radio pulsars (especially the small population of radio pulsars with high inferred magnetic fields; e.g., McLaughlin et al. 2003). Such a connection was previously suggested by the similarity seen in the timing behavior of these two classes of objects, namely the presence of timing noise and glitches (e.g., Kaspi et al. 1999, 2000).

Interestingly, radio emission from XTE J1810-197, which has shown tremendous X-ray brightening, ${ }^{4}$ has only been observed subsequent to its one known X-ray outburst, which occurred sometime between 2002 November and 2003 January (Ibrahim et al. 2004). This indicates that its radio emission is not a stable phenomenon but rather is somehow associated with its X-ray activity. Archival radio pulsation searches prior to this X-ray outburst, in

\footnotetext{
1 Department of Physics and Astronomy, Franklin and Marshall College, Lancaster, PA 17604; fcrawfor@fandm.edu.

2 Department of Physics, McGill University, Montreal, QC H3A 2T8, Canada.

3 Astronomical Institute "Anton Pannekoek," University of Amsterdam, Kruislaan 403, 1098 SJ Amsterdam, The Netherlands.

4 Note that other AXPs have also shown flux variation (e.g., 1E 2259+586; Woods et al. 2004 ), and the most recently established AXP, CXO J164710.2455216 (Muno et al. 2006) had an outburst on 2006 September 21 (Krimm et al. 2006).
}

which the AXP was not detected in radio, show that the pulsed radio flux of XTE J1810-197 increased by more than 1 order of magnitude from its quiescent level (Camilo et al. 2006b). Current radio monitoring (Camilo et al. 2007) reveals that there remains an intrinsic flux variability of about a factor of 2 on day timescales, and that there has been a gradual decrease in brightness and a broadening of the pulse profile since its discovery as a pulsed radio source. Such large-scale intrinsic flux variability has come as a surprise and is not seen in normal radio pulsars (Stinebring et al. 2000). XTE J1810-197 is also peculiar in that it has a very flat radio spectrum and was at the time of its discovery the brightest radio pulsar in the sky above $20 \mathrm{GHz}$.

If the recent emergence of radio emission from XTE J1810197 is indeed directly related to its X-ray behavior, then that may explain why the "persistent" $\mathrm{AXPs}^{5}$ are (at the very least) radio $\mathrm{dim}$. On the other hand, the small beaming fraction of long-period radio pulsars, presumably owing to their large light cylinders and correspondingly small polar caps (e.g., Rankin 1990; Young et al. 1999), and the narrow radio beam of XTE J1810-197 itself (Camilo et al. 2006b) suggest that AXPs, if they are generically radio emitters, should have narrow emission beams and therefore would be unlikely to be detected. In the latter case, that the only confirmed transient AXP is also the only known radio-emitting AXP could be a geometrical coincidence, although it would still be unclear why it was not seen before its X-ray outburst. Only additional radio observations of the AXPs can distinguish between these possibilities and determine the phenomenological link between X-ray and radio activity. Furthermore, given the observed radio flux variability of XTE J1810-197 on both day and year timescales, repeated observations of the AXPs are certainly warranted.

Here we report a sensitive search for radio emission, both periodic and bursting, from three southern AXPs and one southern AXP candidate (potentially also a transient AXP) using the Parkes

\footnotetext{
${ }^{5}$ See the McGill University magnetar catalog at http://www.physics.mcgill.ca/ $\sim$ pulsar/magnetar/main.html.
} 
TABLE 1

Radio Search Parameters and Results

\begin{tabular}{|c|c|c|c|c|}
\hline Parameter & 1E $1048.1-5937$ & AX J1845-0258 & 1E $1841-045$ & 1RXS J170849.0-400910 \\
\hline Spin period (s) & 6.45 & 6.97 & 11.77 & 11.00 \\
\hline 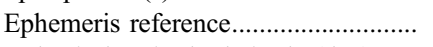 & Kaspi et al. (2001) & Torii et al. $(1998)^{\mathrm{a}}$ & Gotthelf et al. (1999) & Gavriil \& Kaspi (2002) \\
\hline Galactic longitude, latitude (deg) ...... & $288.26,-0.52$ & $29.52,0.07$ & $27.39,-0.01$ & $346.47,0.03$ \\
\hline$T_{\text {sky }}(\mathrm{K})^{\mathrm{b}}$ & 9.1 & 12.3 & 13.2 & 16.3 \\
\hline Observation MJD & 51378 & 51391 & 51382 & 51379 \\
\hline 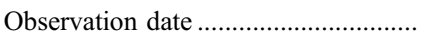 & 1999 Jul 19 & 1999 Aug 1 & 1999 Jul 23 & 1999 Jul 20 \\
\hline$S_{1400}(\mathrm{mJy})^{\mathrm{c}}$ & $\lesssim 0.02$ & $\lesssim 0.02$ & $\lesssim 0.02$ & $\lesssim 0.02$ \\
\hline 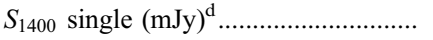 & $\lesssim 875-50$ & $\lesssim 975-60$ & $\lesssim 1000-60$ & $\lesssim 1085-65$ \\
\hline Distance $(\mathrm{kpc}){ }^{\mathrm{e}}$ & $\sim 5 ?$ & $\sim 8 ?$ & $\sim 7$ & $\sim 8 ?$ \\
\hline$L_{1400}\left(\mathrm{mJy} \mathrm{kpc}^{2}\right)^{\mathrm{f}} .$. & $\leq 0.5$ & $\lesssim 1.3$ & $\lesssim 1.0$ & $\lesssim 1.3$ \\
\hline 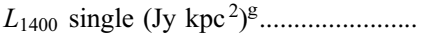 & $\lesssim 22-1.3$ & $\lesssim 62-3.7$ & $\lesssim 49-2.9$ & $\lesssim 69-4.1$ \\
\hline
\end{tabular}

Notes.-Each observation was $16,800 \mathrm{~s}$ long with $0.25 \mathrm{~ms}$ sampling and $288 \mathrm{MHz}$ of bandwidth centered at $1374 \mathrm{MHz}$. These values were used in the sensitivity estimates presented below. Including RFI excision effects in these estimates increases the limits by $\sim 10 \%$.

a There is no period derivative available for this candidate AXP.

b $1374 \mathrm{MHz}$ sky temperature estimated from the model of Haslam et al. (1982) assuming a spectral index of -2.6 .

c $1400 \mathrm{MHz}$ flux density limit on pulsed emission estimated from the modified radiometer equation (Dewey et al. 1985; Lorimer et al. 2006). A pulsed duty cycle of $2.7 \%$ and a $\mathrm{S} / \mathrm{N}$ threshold of 7 was assumed in each case. No red noise contribution due to RFI was included in these estimates.

d Range of single-pulse $1400 \mathrm{MHz}$ flux limits for the range of timescales searched $(0.25-240 \mathrm{~ms})$. No red noise contribution from RFI was included in these estimates.

e Distances were taken from Table 1 of Burgay et al. (2006b), which were based on associations described by Gaensler et al. (2001, 2005). Question marks indicate a significant uncertainty in the actual value owing to different distance estimates by different authors (e.g., Durant \& van Kerkwijk 2006; Kuiper et al. 2006).

f $1400 \mathrm{MHz}$ luminosity limit on pulsed emission. This assumes a beaming fraction of $1 \mathrm{sr}$, so that $L_{1400}=S_{1400} d^{2}$, where $d$ is the best estimate of the AXP distance.

g $1400 \mathrm{MHz}$ luminosity limits on single pulses as determined from the $1400 \mathrm{MHz}$ single-pulse flux limits and the best estimate of the AXP distance (see also the above footnote).

radio telescope. We detect no emission of either kind and provide the most stringent upper limits to date on such emission from these objects. Our results, together with those recently reported by Burgay et al. (2006b) for these same objects, establish that any periodic or bursting radio emission from these sources is either nonexistent, very weak, or very sporadic at the epoch of these observations, as compared with XTE J1810-197, most of the known rotating radio transients (RRATs; McLaughlin et al. 2006), and the bulk of the known radio pulsar population. These null results lay the foundation for future radio observations of these AXPs in the event that one exhibits a significant X-ray outburst like that seen in XTE $\mathrm{J} 1810-197$, as this could in principle establish whether AXP radio emission is associated in some way with X-ray outbursts.

\section{OBSERVATIONS}

Three established AXPs (1E 1048.1-5937, 1E 1841-045, and 1RXS J170849.0-400910) and one AXP candidate (AX J1845-0258) ${ }^{6}$ were observed with the Parkes telescope in 1999 July and August ( Table 1) as part of a larger search campaign to find young, energetic radio pulsars (Crawford et al. 2002). To our knowledge, none of these sources was in a state of X-ray outburst at the time of our observations, although such outbursts are known to be short lived and some may have been missed, despite the X-ray monitoring of these objects (e.g., Kaspi et al. 1999, 2001). Each AXP was observed continuously with a single $16,800 \mathrm{~s}$ $(4.7 \mathrm{hr})$ integration using the center beam of the multibeam receiver (Staveley-Smith et al. 1996; Manchester et al. 2001). This receiver has been successfully used in a number of targeted pulsar searches (e.g., Camilo et al. 2002, 2006a) and large-scale pulsar surveys (Manchester et al. 2001; Edwards et al. 2001; Burgay et al. 2006c). The central observing frequency was $1374 \mathrm{MHz}$

\footnotetext{
${ }^{6}$ AX J1845-0258 is a transient AXP candidate only; see $\S 4$ for further discussion.
}

with $288 \mathrm{MHz}$ of bandwidth, consisting of $963 \mathrm{MHz}$ channels. Each channel was 1 bit sampled every $0.25 \mathrm{~ms}$ and recorded onto DLT magnetic tape. At the start of each observing session, a bright test pulsar (PSR J1359-6038) was observed to verify that the observing system was working properly. Details of the observing setup are similar to those of the Parkes Multibeam Survey (Manchester et al. 2001) and the radio search of these same four AXPs by Burgay et al. (2006b). Although the observations of Burgay et al. (2006b) were conducted with the same observing setup as ours, our observations were longer, analyzed somewhat differently, and, most importantly, taken at a different epoch. The latter point is particularly relevant given the transient nature and day-to-day variability seen in XTE J1810-197.

\section{DATA ANALYSIS AND RESULTS}

Each AXP observation was searched for periodicities, single pulses, and bursts, and was folded at the known neutron star spin period using the X-ray timing ephemeris when available. Standard Fourier-based periodicity searches were reported by Crawford et al. (2002); no AXP counterparts or periodicities indicative of another previously undiscovered pulsar in the same field were found in those searches. Here we describe the single pulse/burst and direct folding analyses, which were conducted with the PRESTO $^{7}$ suite of pulsar analysis tools (Ransom 2001; Ransom et al. 2002).

The first step in both of these analyses was to excise narrowband and transient radio frequency interference (RFI) from the data by identifying bad frequency channels or time intervals and then masking these. Given the slow rotation rates of the AXPs, and the pernicious effect of low-frequency "red" noise in radio data, this step was particularly important for ensuring that a relatively weak signal was not being hidden by RFI. Typically $15 \%-25 \%$

\footnotetext{
7 Available at http://www.cv.nrao.edu/ sransom/presto.
} 
of the frequency channels and $\sim 5 \%$ of the integration time were masked in this process.

\subsection{Folding Search}

In the folding search, the entire $288 \mathrm{MHz}$ of observing bandwidth was subdivided into $329 \mathrm{MHz}$ sub-bands, each of which was transformed to the solar system barycenter and dedispersed at a dispersion measure (DM) of $500 \mathrm{pc} \mathrm{cm}^{-3}$. Each sub-band was folded at the precisely predicted spin period of the AXP at the observation epoch using a contemporaneous ephemeris from $\mathrm{X}$-ray observations (see Table 1 for references; no ephemeris was available for AX J1845-0258 so the discovery period was used). Trial DMs were created by shifting the sub-bands with respect to each other in pulse phase. DMs were searched in spacings of $36-72 \mathrm{pc} \mathrm{cm}^{-3}$ from zero up to a maximum DM of $4000-$ $8000 \mathrm{pc} \mathrm{cm}^{-3}$, depending on the spin period. We also folded the data allowing for a search in period that ranged $\pm 5-10 \mathrm{~ms}$ from the predicted pulse period. In the case of AX J1845-0258, we searched a period range between its discovery period and a period $212 \mathrm{~ms}$ greater than this. The upper limit of this range was determined by using twice the period derivative that results from assuming that AX J1845-0258 has a magnetic field equal to that of SGR J1806-20, the magnetar with the largest inferred field. Separate folds were also made for each target from nine overlapping shorter segments of the full observation. These segments started at intervals of $10 \%$ of the data length and consisted of $20 \%$ of the total integration time. This was done to account for the presence of significant scintillation (unlikely given the high predicted DMs of these sources), strong transient RFI near the AXP spin period, or large-scale intrinsic pulse strength variability on a timescale shorter than the observation length.

We found no convincing periodic signals from any of the AXPs in any of the folds we made. Apart from the statistical significance of the measured $\chi^{2}$ values in the folded profiles, the criteria for differentiating signals potentially related to the AXPs from RFI were that the AXP signal should be broadband, have a steady pulse phase, and be dispersed. None of the observed signals met these criteria, and we therefore associate them with RFI. We note that the frequency decimation of the data into sub-bands, which required an assumed DM, did not introduce a significant amount of dispersive smearing compared to the long spin periods of these neutron stars. Furthermore, dispersive smearing within the frequency channels and multipath scattering effects are not likely to be significant factors in our nondetections: the estimated smearing and scattering times (obtained from the NE2001 Galactic electron model of Cordes \& Lazio [2002] assuming DM values up to $\sim 2000 \mathrm{pc} \mathrm{cm}^{-3}$ ) are less than $1.3 \%$ of the pulse period in each case. This is significantly less than the $2.7 \%$ duty cycle assumed in our sensitivity calculations, which is the duty cycle of XTE J1810-197's integrated radio profile in its discovery paper (see Fig. 1 of Camilo et al. 2006b).

We used the modified radiometer equation presented by Dewey et al. (1985) and more recently by Lorimer et al. (2006) to estimate the $1400 \mathrm{MHz}$ upper limits on the pulsed radio emission from these AXPs. This equation is appropriate to use for the folding searches we conducted (see, e.g., Fig. 2 of Lorimer et al. [2006], which shows the fidelity of using this equation for folding-search sensitivity estimates). The sky temperature at $1374 \mathrm{MHz}$ was estimated for each target AXP using the model of Haslam et al. (1982) and assuming a radio spectral index of -2.6 . As stated above, a duty cycle of $2.7 \%$ was also assumed. The sensitivity limit can easily be computed for different assumed duty cycles in the modified radiometer equation. High-pass filtering is also present in the ob- serving system; this filter has a time constant of $\sim 0.9 \mathrm{~s}$ (Manchester et al. 2001). However, if the radio pulses from our target sources are as narrow as those seen from XTE J1810-197 ( $\leqslant 0.3 \mathrm{~s}$ for all four of our targets, assuming a $2.7 \%$ duty cycle), many harmonics of the fundamental will not suffer any filtering. We therefore do not include any attenuation factor from this filtering in our sensitivity estimates, in contrast to Burgay et al. (2006b). If our assumption is correct, then Burgay et al. (2006b) have underestimated the true sensitivity of their observations by a factor of $2-2.5$. Table 1 lists our calculated upper limits for pulsed radio emission at $1400 \mathrm{MHz}$. It should be noted that these limits do not include loss of data from RFI masking effects and do not account for any red noise contribution from RFI, which is time variable and thus difficult to quantify precisely. Roughly speaking, however, we estimate that the RFI environment may worsen these limits by a factor of 2. We also used the estimated distances to the AXPs to calculate the corresponding $1400 \mathrm{MHz}$ luminosity limits (Table 1).

\subsection{Single Pulse Search}

To search for dispersed single pulses and bursts, the raw data were dedispersed at a set of trial DMs ranging from zero to 4000 $8000 \mathrm{pc} \mathrm{cm}^{-3}$, depending on the spin period. The DM spacing varied as a function of DM (being wider at higher DMs) and was chosen so as not to increase the dispersive smearing already present in the frequency channels. Using the native $0.25 \mathrm{~ms}$ time resolution of the data, each dedispersed time series was searched for candidate single pulses having a signal-to-noise ratio $(\mathrm{S} / \mathrm{N})$ greater than 6.5. This $\mathrm{S} / \mathrm{N}$ threshold was chosen to avoid confusion with the RFI background. To maintain sensitivity to pulses broader than $0.25 \mathrm{~ms}$, matched filtering with a boxcar function of varying widths (ranging from 1 to 30 samples) was also done. ${ }^{8}$ To enhance sensitivity to even longer duration single pulses and bursts, the dedispersed time series were then downsampled by combining the original samples into contiguous blocks of $2,4,8,16$, and 32 samples. Each set of downsampled time series was then searched using the same set of boxcar filters, providing sensitivity to signals of duration $\leqslant 240 \mathrm{~ms}$ (this is equal to the maximum boxcar length, 30 bins, times the maximum downsampled sample time, $8 \mathrm{~ms}$ ). This range encompasses and significantly exceeds the $\lesssim 10 \mathrm{~ms}$ single-pulse widths seen by Camilo et al. (2006b) for XTE J1810-197. The sensitivity to pulses in the downsampled data increases as the square root of the number of combined samples (and further increases with the boxcar filtering), but for each downsampling, a slightly higher $\mathrm{S} / \mathrm{N}$ threshold was used to eliminate spurious candidates from RFI. Specifically, we used S/N thresholds of 7.0, 8.0, 9.0, 11.0, and 12.0 for the downsampling factors described above. To differentiate dispersed single pulses from RFI, results from the different trial DMs were stacked on top of each other in order to identify signals that peaked in $\mathrm{S} / \mathrm{N}$ at a nonzero DM (e.g., Lorimer \& Kramer 2004; McLaughlin et al. 2006). No candidate signals were detected from any of the target AXPs in any of these search trials. Throughout the search, we maintained excellent sensitivity to single pulses for all reasonable DMs.

We have determined single-pulse flux density and luminosity detection limits over the range of pulse timescales we considered (0.25-240 ms), using the modified radiometer equation (e.g., Lorimer et al. 2006) and the $\mathrm{S} / \mathrm{N}$ thresholds described above. These limits are presented in Table 1. As in the case of the folding search, the deleterious effects of red noise and data loss from RFI masking were not included in the sensitivity limits.

\footnotetext{
${ }^{8}$ The boxcar widths used were $1,2,3,4,6,9,14,20$, and 30 samples.
} 


\section{DISCUSSION AND CONCLUSIONS}

We place a flux density limit of $\sim 0.02 \mathrm{mJy}$ on integrated pulsed radio emission from our four AXP targets. This is about 10 times lower than the upper limits placed on XTE J1810-197's pulsed emission in archival Parkes Multibeam Survey observations prior to the outburst of that source (see Table 1 in Camilo et al. 2006b). Our greater sensitivity compared with the Parkes Multibeam Survey data is the result of 8 times longer integration time and higher gain, because our sources were specifically placed at the center of the receiver beam. However, the corresponding $1400 \mathrm{MHz}$ luminosity limits from our observations are only 2-3 times lower than those established for XTE J1810-197 prior to outburst, because that source is estimated to be 2-3 times closer than our target AXPs. The public pulsar catalog (Manchester et al. 2005) ${ }^{9}$ shows that of the known pulsars with measured $1400 \mathrm{MHz}$ radio luminosities, $\sim 5 \%$ have values below $1 \mathrm{mJy} \mathrm{kpc}^{2}$, which is comparable to our limits. Based on the work of Lyne et al. (1998), Gaensler et al. (2001) estimate that $\gtrsim 60 \%$ of potentially observable pulsars have $1400 \mathrm{MHz}$ luminosities less than $1 \mathrm{mJy} \mathrm{kpc}^{2}$. It is therefore conceivable that weak radio pulses could be emitted by our targets but that they are below our detection threshold.

However, the average $1400 \mathrm{MHz}$ flux density reported by Camilo et al. (2006b) for XTE J1810-197 soon after it was first detected was $\sim 8 \mathrm{mJy}$. This corresponds to a $1400 \mathrm{MHz}$ luminosity of $\sim 80 \mathrm{mJy} \mathrm{kpc}{ }^{2}$, which is almost 2 orders of magnitude larger than the luminosity limits of our searches. Thus, comparably strong radio emission from our target AXPs would have been easily detectable if it were beamed in our direction. Long-period pulsars typically have smaller duty cycles and narrower emission beams than faster pulsars (Young et al. 1999), thereby making line-ofsight detection more unlikely. It is possible that all of our target AXPs could be strong radio emitters but that none is detectable by us owing to unfavorable viewing geometries. We note, however, that the emission from XTE J1810-197 is different than for normal rotation-powered radio pulsars. Camilo et al. (2006b) argue that it is possible that the radio emission from XTE J1810197 is generated on closed field lines (as opposed to conventional radio pulsar emission, which streams along open magnetic field lines above the magnetic poles) and beamed into a much broader range of angles. If radio emission from magnetars is emitted in a variety of directions, then detectability is less contingent on beaming.

One of the sources in our sample, AX J1845-0258, is a candidate transient AXP having had an X-ray outburst in 1993 (Gotthelf \& Vasisht 1998; Torii et al. 1998) but not having been seen to emit pulsed X-ray emission since then. In this sense, it may be like XTE J1810-197, but in a quiescent state in 1999, when the observations reported here were taken (see Tam et al. [2006] for a more detailed summary of this source's X-ray behavior). If AX J1845-0258 is indeed an AXP and undergoes another flare in the future, it will be important to compare any subsequently detected radio emission with that seen from XTE J1810-197 subsequent to its X-ray outburst. In that event, the upper limits presented here during AX J1845-0258's quiescent phase will be a critical piece of information. The observation of an outburst on 2006 September 21 (Krimm et al. 2006) from the most recently established AXP, CXO J164710.2-455216 (Muno et al. 2006; Woods et al. 2006), suggests that this outburst was similar to the

\footnotetext{
9 Available at http://www.atnf.csiro.au/research/pulsar/psrcat.
}

one seen from XTE J1810-197. No pulsed radio emission was detected a week after the burst, to a limit of $0.04 \mathrm{mJy}$ (Burgay et al. 2006a; further observations are planned).

For a comparison of our single-pulse upper limits with the observed single pulses reported for XTE J1810-197 by Camilo et al. (2006b), we compared the corresponding single-pulse luminosity limits for our sources and for XTE J1810-197 (Table 1). Our luminosity limits in the most conservative case range from 22 to $69 \mathrm{Jy} \mathrm{kpc}^{2}$, below the luminosity of $\gtrsim 100 \mathrm{Jy} \mathrm{kpc}^{2}$ derived from the $\gtrsim 10$ Jy pulses for XTE J1810-197 reported by Camilo et al. (2006b). Single pulses were also detected from almost every rotation of XTE J1810-197. This suggests that we would have expected to detect a large number of pulses during the course of our observations if comparable radio emission were being emitted by our targets.

Our nondetection of single pulses further weakens the hypothesis that some of the sporadically emitting radio sources known as RRATs (McLaughlin et al. 2006) are magnetars. This link has also been weakened by two other recent results. First, the discovery of X-ray emission associated with the most "magnetar-like" of the RRATs, J1819-1458, shows that this emission is more typical of middle-aged rotation-powered pulsars than that seen from the magnetars (Reynolds et al. 2006). Secondly, Weltevrede et al. (2006) argue that the nearby rotation-powered pulsar PSR B0656+14 shows emission that would cause it to be identified as an RRAT if its distance were greater. We note, however, that single radio pulse/ burst searches toward the AXPs are also motivated for other reasons. XTE J1810-197 has shown strong individual pulses, with an intensity distribution that follows a power law. This presents the possibility of detecting individual bright pulses even if the majority of the radio emission is below the detection threshold. If magnetars can emit giant pulses, this is also potentially interesting, as such pulses may be generated in a different part of the magnetosphere than "normal" pulses (Knight et al. 2006 and references therein). If beaming plays an important role in the detectability of radio emission from magnetars, then giant pulses could provide another window in which to detect radio emission. Furthermore, AXPs are known to emit rare X-ray bursts. One of the AXPs in our sample, 1E 1048.1-5937, was the first shown to have such behavior (Gavriil et al. 2002). Such X-ray bursts may be accompanied by radio bursts (Lyutikov 2002).

In conclusion, our nondetections support the hypothesis that pulsed radio emission from AXPs is connected to X-ray outburst activity, as may be the case for XTE J1810-197. However, unfavorable viewing geometries and very weak radio emission cannot be dismissed as reasons for our nondetections. Given that the transient radio emission observed from XTE J1810-197 appears to be linked with its transient X-ray behavior, continued radio monitoring of all the AXPs is warranted, particularly after periods of X-ray burst activity.

We thank Scott Ransom for creating the PRESTO suite of software tools, which were used in this analysis, and we acknowledge use of the McGill University magnetar catalog. J. W. T. H. holds an NSERC Postdoctoral Fellowship. V. M. K. is the Lorne Trottier Canada Research Chair and acknowledges support from NSERC, CIAR, and FQRNT. The Parkes radio telescope is part of the Australia Telescope, which is funded by the Commonwealth of Australia for operation as a National Facility managed by CSIRO. 


\section{REFERENCES}

Burgay, M., Rea, N., Israel, G., \& Possenti, A. 2006a, ATel, 903

Burgay, M., Rea, N., Israel, G. L., Possenti, A., Burderi, L., di Salvo, T., D'Amico, N., \& Stella, L. 2006b, MNRAS, 372, 410

Burgay, M., et al. 2006c, MNRAS, 368, 283

Camilo, F., Manchester, R. N., Gaensler, B. M., Lorimer, D. L., \& Sarkissian, J. 2002, ApJ, 567, L71

Camilo, F., Ransom, S. M., Gaensler, B. M., Slane, P. O., Lorimer, D. R., Reynolds, J., Manchester, R. N., \& Murray, S. S. 2006a, ApJ, 637, 456

Camilo, F., Ransom, S. M., Halpern, J. P., Reynolds, J., Helfand, D. J., Zimmerman, N., \& Sarkissian, J. 2006b, Nature, 442, 892

Camilo, F., et al. 2007, ApJ, 663, 497

Cordes, J. M., \& Lazio, T. J. W. 2002, preprint (astro-ph/0207156)

Crawford, F., Pivovaroff, M. J., Kaspi, V. M., \& Manchester, R. N. 2002, in ASP Conf. Ser. 271, Neutron Stars in Supernova Remnants, ed. P. Slane \& B. Gaensler (San Francisco: ASP), 37

Dewey, R. J., Taylor, J. H., Weisberg, J. M., \& Stokes, G. H. 1985, ApJ, 294 , L25

Durant, M., \& van Kerkwijk, M. H. 2006, ApJ, 650, 1070

Edwards, R. T., Bailes, M., van Straten, W., \& Britton, M. C. 2001, MNRAS, 326,358

Gaensler, B. M., McClure-Griffiths, N. M., Oey, M. S., Haverkorn, M., Dickey, J. M., \& Green, A. J. 2005, ApJ, 620, L95

Gaensler, B. M., Slane, P. O., Gotthelf, E. V., \& Vasisht, G. 2001, ApJ, 559, 963

Gavriil, F. P., \& Kaspi, V. M. 2002, ApJ, 567, 1067

Gavriil, F. P., Kaspi, V. M., \& Woods, P. M. 2002, Nature, 419, 142

Gotthelf, E. V., \& Vasisht, G. 1998, NewA, 3, 293

Gotthelf, E. V., Vasisht, G., \& Dotani, T. 1999, ApJ, 522, L49

Halpern, J. P., Gotthelf, E. V., Becker, R. H., Helfand, D. J., \& White, R. L. 2005, ApJ, 632, L29

Haslam, C. G. T., Stoffel, H., Salter, C. J., \& Wilson, W. E. 1982, A\&AS, 47, 1 Ibrahim, A. I., et al. 2004, ApJ, 609, L21

Israel, G. L., Mereghetti, S., \& Stella, L. 2002, Mem. Soc. Astron. Italiana, 73, 465

Kaspi, V. M. 2007, Ap\&SS, in press (astro-ph/0610304)

Kaspi, V. M., Chakrabarty, D., \& Steinberger, J. 1999, ApJ, 525, L33

Kaspi, V. M., Gavriil, F. P., Chakrabarty, D., Lackey, J. R., \& Muno, M. P. 2001, ApJ, 558, 253
Kaspi, V. M., Lackey, J. R., \& Chakrabarty, D. 2000, ApJ, 537, L31

Knight, H. S., Bailes, M., Manchester, R. N., Ord, S. M., \& Jacoby, B. A. 2006 ApJ, 640, 941

Krimm, H., Barthelmy, S., Campana, S., Cummings, J., Israel, G., Palmer, D., \& Parsons, A. 2006, GCN Circ. 5581 (http://gcn.gsfc.nasa.gov/gen/gcn3/ 5581.gen 3 )

Kuiper, L., Hermsen, W., den Hartog, P. R., \& Collmar, W. 2006, ApJ, 645, 556

Lorimer, D. R., \& Kramer, M. 2004, Handbook of Pulsar Astronomy (Cambridge: Cambridge Univ. Press)

Lorimer, D. R., et al. 2006, MNRAS, 372, 777

Lyne, A. G., et al. 1998, MNRAS, 295, 743

Lyutikov, M. 2002, ApJ, 580, L65

Manchester, R. N., Hobbs, G. B., Teoh, A., \& Hobbs, M. 2005, AJ, 129, 1993

Manchester, R. N., et al. 2001, MNRAS, 328, 17

McLaughlin, M. A., et al. 2003, ApJ, 591, L135 2006, Nature, 439, 817

Muno, M. P., et al. 2006, ApJ, 636, L41

Rankin, J. M. 1990, ApJ, 352, 247

Ransom, S. M. 2001, Ph.D. thesis, Harvard Univ

Ransom, S. M., Eikenberry, S. S., \& Middleditch, J. 2002, AJ, 124, 1788

Reynolds, S. P., et al. 2006, ApJ, 639, L71

Staveley-Smith, L., et al. 1996, Publ. Astron. Soc. Australia, 13, 243

Stinebring, D. R., Smirnova, T. V., Hankins, T. H., Hovis, J., Kaspi, V., Kempner, J., Meyers, E., \& Nice, D. J. 2000, ApJ, 539, 300

Tam, C. R., Kaspi, V. M., Gaensler, B. M., \& Gotthelf, E. V. 2006, ApJ, 652, 548

Torii, K., Kinugasa, K., Katayama, K., Tsunemi, H., \& Yamauchi, S. 1998, ApJ, 503, 843

Weltevrede, P., Stappers, B. W., Rankin, J. M., \& Wright, G. A. E. 2006, ApJ, 645, L149

Woods, P. M., Kaspi, V. M., \& Gavriil, F. P. 2006, ATel, 929

Woods, P. M., \& Thompson, C. 2006, in Compact Stellar X-ray Sources, ed. W. H. G. Lewin \& M. van der Klis (Cambridge: Cambridge Univ. Press), 547 Woods, P. M., et al. 2004, ApJ, 605, 378

Young, M. D., Manchester, R. N., \& Johnston, S. 1999, Nature, 400, 848 